\title{
Effect of prehospital initiation of therapeutic hypothermia in adults with cardiac arrest on time-to-target temperature
}

\author{
Eric M. Schenfeld, MD*; Jonathan Studnek, $\mathrm{PhD}^{\dagger}$; Alan C. Heffner, MD§; Marcy Nussbaum, MS"; \\ Kathi Kraft" ; David A. Pearson, MD
}

\section{ABSTRACT}

Objective: Despite growing adoption, the impact of prehospital initiation of therapeutic hypothermia on outcomes of cardiac arrest patients is unknown. The objective of this study was to determine if prehospital administration of cold intravenous fluids improved the time-to-target temperature. Methods: All patients enrolled in an institutional postcardiac arrest treatment pathway were prospectively registered into a quality assurance database. Patients undergoing cooling induction on hospital arrival were compared to those receiving a new treatment protocol initiated during the study period involving prehospital cooling with $4^{\circ} \mathrm{C}\left(39.2^{\circ} \mathrm{F}\right)$ normal saline. The primary outcome was the time-to-target temperature. Secondary outcomes included emergency medicine system transport time metrics, mortality, and neurologic status at discharge and 1 year.

Results: One hundred thirty-two patients were enrolled during the study period. The initial rhythm was ventricular fibrillation/tachycardia in $63 \%$ and asystole/pulseless electrical activity in $36 \%$. Eighty patients received prehospital cooling and 52 patients did not and comprised the historical control group. Time-to-target temperatures were not significantly different between prehospital and hospital cooled groups (256 v. 271 minutes, respectively, $p=0.64$ ), nor was there any improvement in hospital survival $(54 \%$ v. $50 \%, p=$ $0.67)$, good neurologic outcome ( $49 \%$ v. $44 \%, p=0.61$ ), or $1-$ year survival $(49 \%$ v. $42 \%, p=0.46)$ between the two groups. Transport times were longer in the prehospital cooled group. Conclusions: Out-of-hospital cardiac arrest patients treated with prehospital cooling before arrival at our urban hospital did not have faster time-to-target temperature or improvement in outcomes compared to patients cooled immediately on emergency department arrival. Further research is needed to determine if any benefits exist from prehospital cooling prior to its widespread adoption.

\section{RÉSUMÉ}

Objectif: Bien que I'amorce de I'hypothermie thérapeutique en phase préhospitalière soit de plus en plus répandue, on n'en connaît pas l'effet sur les résultats, chez les patients victimes d'un arrêt cardiaque. L'étude visait à déterminer si l'administration intraveineuse de liquides froids, en phase préhospitalière, permettait $d^{\prime}$ 'atteindre plus rapidement la température cible.

Méthode: Tous les patients soumis à un parcours de traitement, en établissement, pour un arrêt cardiaque ont été inscrits de manière prospective dans une base de données sur l'assurance de la qualité. Les patients soumis au refroidissement à leur arrivée à l'hôpital ont été comparés avec ceux soumis au nouveau protocole de traitement mis en œuvre durant la période à l'étude, comportant un refroidissement préhospitalier à l'aide de l'administration d'une solution physiologique salée maintenue à $4^{\circ} \mathrm{C}\left(39.2^{\circ} \mathrm{F}\right)$. Le principal critère d'évaluation était le temps nécessaire à I'atteinte de la température cible. Les critères d'évaluation secondaires comprenaient les mesures du temps de transport médical d'urgence, la mortalité, l'état neurologique au moment du congé et au bout de 1 an.

Résultats: Cent trente-deux patients ont été inscrits durant la période à l'étude. Les rythmes enregistrés au départ étaient la fibrillation ou la tachycardie ventriculaires dans $63 \%$ des cas ou encore l'asystole ou une activité électrique non pulsatile dans $36 \%$ des cas. Quatre-vingt patients ont été soumis au refroidissement préhospitalier et 52 patients, formant le groupe témoin historique, ne l'ont pas été. Le temps nécessaire à l'atteinte de la température cible n'était

From *Utah Emergency Physicians, Department of Emergency Medicine, Intermountain Medical Center, Intermountain Healthcare, Salt Lake City, UT; †Mecklenburg EMS Agency, Charlotte, NC; ‡Division of Critical Care Medicine, Department of Internal Medicine, Carolinas Medical Center, Charlotte, NC; §Department of Emergency Medicine, Carolinas Medical Center, Charlotte, NC; and ॥Carolinas Health Care System, Dickson Advanced Analytics Group, Charlotte, NC.

Correspondence to: Dr. Eric M. Schenfeld, Utah Emergency Physicians, Intermountain Healthcare, PO Box 571117, Murray, UT 84157-1117; emschenfeld@gmail.com; david.pearson@carolinashealthcare.org.

This article has been peer reviewed.

(c) Canadian Association of Emergency Physicians 
pas sensiblement différent entre le groupe de refroidissement préhospitalier et le groupe de refroidissement hospitalier (256 minutes contre [c.] 271, respectivement; $p=0.64$ ), pas plus qu'il n'y avait d'amélioration en ce qui concerne la survie à I'hôpital ( $54 \%$ c. $50 \% ; p=0.67$ ), I'état neurologique ( $49 \%$ c. $44 \% ; p=0.61$ ) et la survie au bout de 1 an (49\% c. $42 \% ; p=0.46$ ) entre les deux groupes. Le temps de transport était toutefois plus long dans le groupe de refroidissement préhospitalier.

Conclusions: Le refroidissement préhospitalier chez les patients ayant subi un arrêt cardiaque, avant l'arrivée dans un hôpital urbain, n'a pas permis d'atteindre plus rapidement la température cible ou d'améliorer les résultats comparativement aux patients soumis au refroidissement dès leur arrivée au service des urgences. Aussi une recherche approfondie s'impose-t-elle afin de déterminer si le refroidissement préhospitalier comporte quelque avantage, et ce, avant l'adoption élargie de la pratique.

Keywords: cardiac arrest, prehospital medicine, therapeutic hypothermia
The Resuscitation Outcomes Consortium estimates that there are 359,000 out-of-hospital cardiac arrests (OOHCAs) in the United States each year. ${ }^{1}$ Following two landmark studies in 2002, therapeutic hypothermia $(\mathrm{TH})$ has become standard treatment for patients who remain comatose following return of spontaneous circulation (ROSC) after cardiac arrest. ${ }^{2,3}$ Observational experiences have confirmed the feasibility of cooling, and there has been consensus across guidelines that cooling initiation following ROSC is beneficial to both mortality and neurologic outcomes. ${ }^{4,5}$

The optimal timing of therapeutic cooling remains unclear. Animal models have demonstrated that immediate and faster cooling yields improved outcomes, ${ }^{6-8}$ but other studies have suggested that no benefit exists in short-term survival with rapid cooling, even when compared to normothermic postresuscitation care. ${ }^{8}$ Observational human studies, although limited by their design, have also yielded conflicting results, with rapid cooling reported to be associated with improved, neutral, and negative effects on neurologic status and mortality. ${ }^{9-15}$

Despite the lack of clear evidence, many prehospital systems have implemented prehospital cooling protocols for post-cardiac arrest patients who remain comatose in the field. The benefit, if any, of this practice is unknown. The purpose of our study was to assess the impact of prehospital cooling on process measures and patient outcomes. Our primary objective was to determine if prehospital administration of cold intravenous (IV) fluids improved the time-totarget temperature. For secondary purposes, we analyzed the effect of prehospital cooling on mortality at hospital discharge and 1 year, neurologic outcome, and emergency medical system (EMS) time metrics.

\section{METHODS}

\section{Study design and setting}

We performed a retrospective analysis on all patients treated in the $\mathrm{TH}$ clinical pathway of Carolinas Medical Center, an urban, 900-bed teaching hospital, from November 2007 through November 2011. This was a before-and-after study, with the intervention being initiation of prehospital cooling, whereas the historical control group received no prehospital cooling. Our institution is a regional cardiac resuscitation centre that serves a network of 25 referring hospitals in the region and an ST elevation myocardial infarction (STEMI) receiving centre as designated by the American Heart Association Mission: Lifeline systems of care program. In April 2009 (approximately midway through our enrolment period), our local prehospital EMS agency implemented a protocol for prehospital cooling of patients experiencing OOHCA.

The EMS agency under study, Mecklenburg EMS Agency, is a municipal system serving a population of approximately 867,000 . Over the study period, its average annual call volume was 90,000 , resulting in approximately 69,000 patient transports. Patients were transported to any of the seven metropolitan hospitals of a single academic institution and a separate regional tertiary care facility. All ambulances were staffed with at least one paramedic and one emergency medical technician (EMT)-basic. First responders within the city and county were trained at the EMT-basic level and had access to an automated external defibrillator (AED). Prehospital triage, treatment, and transport protocols were uniform within both the county and the city limits.

Our protocol for prehospital cooling has been described previously. ${ }^{16}$ Briefly, prehospital providers initiated infusion of cooled normal saline $\left(4^{\circ} \mathrm{C}\right.$ 
$\left[39.2^{\circ} \mathrm{F}\right]$ ) via the first established IV or intraosseous access for all nontraumatic cardiac arrest victims regardless of their initial rhythm. This included patients undergoing active resuscitation for cardiac arrest or following ROSC, with the goal of earliest possible initiation of cooling. Infusions were given as $500 \mathrm{~mL}$ rapid IV bolus aliquots by gravity infusion. Patients could receive repeated rounds of infusion up to a maximum of $2 \mathrm{~L}$ of fluid. Ambulances were equipped with refrigerators that maintained $1 \mathrm{~L}$ normal saline bags at $4^{\circ} \mathrm{C}$. All other patient care was in accordance with Advanced Cardiac Life Support guidelines. Prehospital providers did not administer paralytics or sedatives and had instructions to discontinue TH if shivering developed.

Patients receiving prehospital $\mathrm{TH}$ served as the intervention group of this analysis and were compared to patients enrolled prior to the implementation of a prehospital cooling protocol. Our historical controls thus had initiation of TH in the emergency department (ED). The in-hospital cooling protocol was standardized for both the intervention and the control group, and there were no changes to the in-hospital protocol during the study period. This protocol included placement of ice packs to the groin, axilla, and neck; continuation of cooled IV fluid until a total of $30 \mathrm{~mL} / \mathrm{kg}$ was administered; and implementation of the CR Bard (formerly Medivance, Inc., Louisville, CO) Arctic Sun 2000 cooling device set to achieve a target temperature of $33^{\circ} \mathrm{C}\left(91.4^{\circ} \mathrm{F}\right)$ as rapidly as possible.

This investigation was a retrospective analysis of an existing database. Clinical data, including arrest and treatment variables, complications, and outcomes, were prospectively collected on consecutive patients through the use of a preformatted standard data collection tool using Utstein criteria. ${ }^{17}$ All data elements were chart abstracted and underwent both concurrent and retrospective review. These elements remained consistent throughout the study period and were defined using a "data dictionary." All data were manually entered from the electronic medical records and EMS records into a Web-based data collection tool. No interrater reliability assessment of the data elements was performed. The institutional review and privacy board of Carolinas Medical Center approved this study.

\section{Study subjects}

Patients experiencing a cardiac arrest of suspected cardiac etiology, regardless of presenting rhythm, received initiation of prehospital TH. Only those arriving directly at our centre via ground prehospital transport were eligible for study inclusion; patients transferred from outlying hospitals were excluded. Patients eligible for our hospital TH protocol were identified in the ED following arrival. Resuscitated victims of nontraumatic cardiac arrest with persistent coma (defined as a Glasgow Coma Scale [GCS] score $\leq 8$ and/or unable to follow verbal commands) for at least 15 minutes following ROSC were eligible. The guideline emphasized the evidence for a benefit from therapeutic cooling in patients suffering cardiac arrest with an initial rhythm of ventricular tachycardia or ventricular fibrillation. However, all nontraumatic patients were eligible for the clinical pathway regardless of their initial arrest rhythm. Our guideline recommended strong consideration of cooling for patients suffering arrest with an initial rhythm of pulseless electrical activity or asystole if the time of arrest to ROSC was less than 30 minutes. Patients were included in the analysis if they survived to reach the intensive care unit (ICU) with intent to complete the cooling pathway. In the setting of arrests with an initial rhythm other than ventricular tachycardia or ventricular fibrillation, the decision to enrol into the cooling pathway was made after a joint discussion between the attending emergency physician and the critical care physician.

Absolute contraindications for pathway implementation included a valid do not resuscitate order or known severe terminal illness preceding the cardiac arrest. Relative contraindications included pregnancy, encephalopathy suspected to be unrelated to cerebral anoxia (e.g., overdose, intoxication, intracranial hemorrhage, stroke, or trauma), active hemorrhage, severe systemic infection, moribund cardiovascular status or severe shock refractory to medical interventions, arrest duration greater than 60 minutes, and arrest to cooling initiation interval greater than 6 hours. Clinical discretion was emphasized and superseded the relative contraindications if the perceived potential benefit of therapy outweighed the risk. Continuation of $\mathrm{TH}$ initiated in the prehospital setting was not mandatory and occurred at the discretion of treating physicians on arrival at our centre.

\section{Data analysis}

We designed our study to have $90 \%$ power to detect a $10 \%$ absolute difference in time-to-target temperature, 


\begin{tabular}{|c|c|c|c|c|}
\hline & Total & Prehospital cooling & No prehospital cooling & \\
\hline Characteristic & $(N=132)$ & $(n=80)$ & $(n=52)$ & $p$ value \\
\hline Age, mean years $\pm S D$ & $58.2 \pm 14.0$ & $58.4 \pm 12.8$ & $57.8 \pm 15.8$ & 0.84 \\
\hline Male gender, $n(\%)$ & $82(62)$ & $52(65)$ & $30(58)$ & 0.40 \\
\hline Witnessed arrest, $n(\%)$ & $111(84)$ & $65(81)$ & $46(89)$ & 0.27 \\
\hline Bystander CPR, $n(\%)$ & $94(75)$ & $65(81)$ & $29(63)$ & 0.02 \\
\hline Field AED use, $n(\%)$ & $53(40)$ & $38(48)$ & $15(29)$ & 0.03 \\
\hline Time from arrest to ROSC* (min) & $19(12,29)$ & $20(14,27)$ & $17(8,30)$ & 0.14 \\
\hline \multicolumn{5}{|l|}{ Initial arrest rhythm, n (\%) } \\
\hline VTNF & $83(63)$ & $50(63)$ & $33(64)$ & 0.52 \\
\hline PEA & $28(21)$ & $15(19)$ & $13(25)$ & \\
\hline Asystole & $20(15)$ & $14(18)$ & $6(12)$ & \\
\hline Best GCS score prior to $\mathrm{TH}^{*}$ & $3(3,3)$ & $3(3,3)$ & $3(3,4)$ & 0.47 \\
\hline STEMI at presentation & $11(8.3 \%)$ & $6(8 \%)$ & $5(10 \%)$ & 0.75 \\
\hline
\end{tabular}

based on the mean and standard deviation (SD) for the population under study. Our primary outcome was the time-to-target temperature following achievement of ROSC. Secondary outcomes were survival to hospital discharge, 1-year mortality, neurologic outcome as measured by the Pittsburgh Cerebral Performance Category, ${ }^{17}$ and process of care measures focusing on transport times. Cerebral functional status was determined at hospital discharge, and Pittsburgh Cerebral Performance Category 1 or 2 was deemed to be a good neurologic outcome. ${ }^{2}$ All patients admitted to the ICU with intention to undergo $\mathrm{TH}$ were included in the analysis, regardless of the extent to which the cooling protocol was followed or completed. Health care system electronic health records and the Social Security Death Index were queried to determine the survival of subjects at 1-year postarrest.

For the analysis, categorical variables were compared with the chi-square test or, in the case of small counts, Fisher exact tests. Two-sample $t$-tests and Wilcoxon rank sum tests were used for continuous variables as appropriate, based on the distribution of the data. Two-sided $p$ values less than 0.05 were considered statistically significant. One-year survival was assessed using Kaplan-Meier estimates and a logrank test. A secondary analysis was performed using an

\begin{tabular}{|c|c|c|c|c|}
\hline & Total & Prehospital cooling & No prehospital cooling & \\
\hline & $(N=132)$ & $(n=80)$ & $(n=52)$ & $p$ value \\
\hline $\begin{array}{l}\text { Prehospital fluids initiated before ROSC, } \\
n(\%)\end{array}$ & $59(48)$ & $57(74)$ & $2(4)$ & $<0.001$ \\
\hline Achievement of target temperature, $n(\%)$ & 131 (99) & 79 (99) & $52(100)$ & $>0.99$ \\
\hline Time from arrest to hospital arrival* & $40(33,49)$ & $44(37,53)$ & $34(19,41)$ & $<0.001$ \\
\hline $\begin{array}{l}\text { Time of initiation of cold fluids to hospital } \\
\text { arrival* }^{*}\end{array}$ & $15(-13,32)$ & $30(19,38)$ & $-22(-75,-10)$ & $<0.001$ \\
\hline Time of ROSC to hospital arrival* & $18(5,30)$ & $20(13,32)$ & $12(0,23)$ & 0.005 \\
\hline Time from EMS arrival to hospital arrival* & $34(26,42)$ & $38(30,43)$ & $26(22,35)$ & $<0.001$ \\
\hline Time from collapse to initiation of cooling* & $26(14,59)$ & $16(11,21)$ & $66(38,110)$ & $<0.001$ \\
\hline ROSC to target temperature* & $257(184,349)$ & $256(185,341)$ & $271(184,369)$ & 0.64 \\
\hline
\end{tabular}




\begin{tabular}{|c|c|c|c|c|}
\hline & Hospital survival & & Good neurologic outcome & \\
\hline Outcome & $(n=69)$ & $p$ value & $(n=62)$ & $p$ value \\
\hline Prehospital cooling, $n(\%)$ & $43(54)$ & 0.67 & $39(49)$ & 0.61 \\
\hline No prehospital cooling, $n(\%)$ & $26(50)$ & & $23(44)$ & \\
\hline \multicolumn{5}{|l|}{ Time of fluid initiation } \\
\hline Initiated pre-ROSC ( $n=59), n(\%)$ & $28(48)$ & 0.35 & $25(42)$ & 0.39 \\
\hline Initiated post-ROSC $(n=64), n(\%)$ & $36(56)$ & & $32(50)$ & \\
\hline
\end{tabular}

unadjusted Cox proportional hazards model to determine if differences existed between the prehospital cooling and the in-hospital cooling groups. This study was not powered to detect a difference in mortality. All analyses were conducted using $S A S$ statistical software version 9.2 (SAS Institute, Cary, NC).

\section{RESULTS}

One hundred thirty-two OOHCA patients were enrolled during the study period: 80 who received prehospital cooling and 52 who did not and had TH initiated in the ED. Baseline participant characteristics are shown in Table 1. Patients in the prehospital cooling group were more likely to have received bystander cardiopulmonary resuscitation (CPR) and field AED use.

Process of care metrics for the two groups are provided in Table 2. Of those who were administered cold IV fluids prehospital, $31 \%$ received $<500 \mathrm{~mL}$, $46 \%$ received 500 to $1,000 \mathrm{~mL}, 12 \%$ received 1,001 to $1,500 \mathrm{~mL}$, and $11 \%$ received 1,501 to $2,000 \mathrm{~mL}$.
There was no difference in time-to-target temperature between the groups receiving and not receiving prehospital cooling (256 [95\% CI 185-341] and 271 [95\% CI 184-369] minutes, respectively, $p=0.64$ ). Transport intervals for patients receiving prehospital TH were all longer than those receiving prehospital TH. In contrast, time from collapse to initiation of cooling was longer in patients cooled on hospital arrival compared to those cooled prehospital.

Clinical outcomes are provided in Table 3. There was no statistically significant difference found in hospital survival or good neurologic outcomes in the two groups. A subgroup comparison of the patients who received initiation of cold IV fluids prior to ROSC (i.e., intra-arrest initiation) with those who received initiation following ROSC similarly found no significant difference in clinical outcomes.

Figure 1 provides data for survival at 1 year and shows that there was no statistically significant difference between the groups. Data were available for 128 patients; the 4 censored patients were alive but had not reached the 1-year threshold at the time of the analysis.

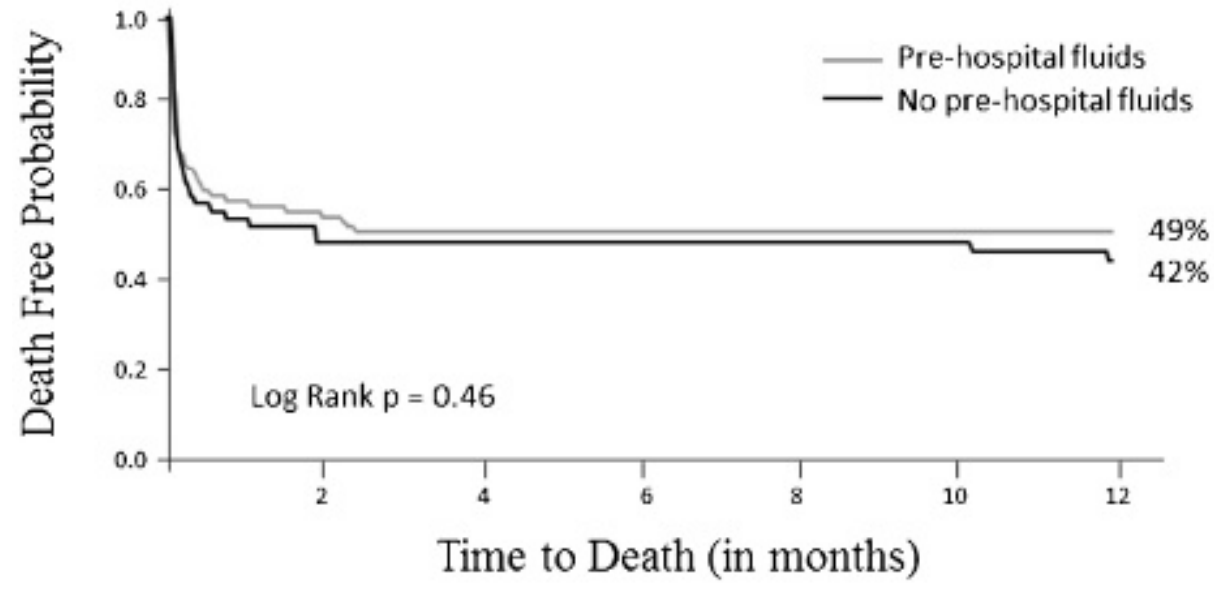

Figure 1. One-year mortality. 


\section{DISCUSSION}

Our results suggest that prehospital implementation of therapeutic cooling expedites TH induction but is not associated with improved time-to-target temperature and results in prehospital transport delays. Our study adds to the evidence on prehospital cooling by suggesting that simply implementing the earliest possible postarrest cooling regimen, via intra-arrest cooling or immediately postarrest, does not necessarily result in achievement of a faster time-to-goal temperature.

Post-cardiac arrest TH is a powerful treatment that is known to impact survival and neurologic outcome and has been widely emphasized over the past decade. The optimal method, timing, rate, and period of $\mathrm{TH}$ are unclear and represent a prime target of investigation to improve therapeutic effect.

Although our findings may arise from the inherent challenges of cooling in the prehospital environment, it is noteworthy that we also found a longer period of prehospital care among those patients who received prehospital cooling. We suspect that a contributor to the prolonged EMS intervals we observed was the implementation of a prehospital "focused CPR" protocol several months prior to initiation of the TH protocol. This protocol emphasized minimally interrupted chest compressions and remaining on the scene of an arrest for a minimum of 20 minutes or until ROSC was achieved. When this protocol is considered in the context of the increased bystander CPR and field AED use in patients who received prehospital cooling, it is possible that these interventions may have either contributed to the observed delayed time to hospital arrival or led to an increased propensity to continue the cooling protocol inhospital once such patients arrived. Previous research suggests that the delay this protocol resulted in may not have any bearing on survival outcomes. ${ }^{18,19}$ It is clear that early initiation of cooling is not the sole factor in achieving goal temperature and possible that delays arising from patient sedation, paralysis, deviation from hospital protocol, and additional $\mathrm{TH}$ induction measures impacted our results. Our centre's post-cardiac arrest clinical pathway is composed of a bundle of therapeutic interventions in addition to TH. The prehospital delays present in our intervention group may be a surrogate for delays in other important hospital-based interventions, including cardiopulmonary stabilization and percutaneous coronary intervention.
Despite the lack of benefit in terms of time-to-target temperature, survival, and neurologic outcome, in our study population, our findings do not completely exclude the possibility of benefit from prehospital cooling. Although not studied, the initiation of prehospital cooling could prevent hyperthermia despite not leading to a faster time-to-target temperature, which could be protective to the injured brain. The therapeutic momentum of initiating cooling in the field could also lead to increased overall completion of the entire therapeutic cooling pathway and more aggressive overall care. The determination of whether prehospital cooling offers benefit requires further assessment investigating a variety of parameters.

Data on the impact of early TH are confusing and conflicted. Animal studies have suggested that early cooling improves ROSC and outcomes. ${ }^{6,8}$ Based on the premise of time sensitivity of cooling for neuroprotection, many EMS systems have adopted prehospital TH induction to expedite cooling. Prehospital induction via $4^{\circ} \mathrm{C}$ normal saline is feasible, safe, and effective in decreasing core temperature. ${ }^{11,13}$ However, four prospective randomized trials have found no difference in outcomes with prehospital cooling initiation. ${ }^{12,14,20,21}$ In contrast, a retrospective study using ice packs found deleterious effects of every 5-minute delay in $\mathrm{TH}$ initiation and every 30-minute delay in time-to-target temperature. ${ }^{22}$ Rapid cooling has been associated with adverse outcomes thought to be linked to more severely impaired thermoregulation in those with severe brain injury. ${ }^{10,23}$ Adjustment for admission body temperature appears to negate the signal arising from analyses of this issue. ${ }^{18,20}$ Garrett and colleagues suggested potential improved ROSC rates when intra-arrest cooling was used..$^{23}$ Taken in its entirety, the body of literature regarding the optimal timing of $\mathrm{TH}$ induction remains unclear. Our study adds to the current literature by demonstrating that prehospital cooling with IV fluids does not shorten the time-to-target temperature.

\section{LIMITATIONS}

A number of important limitations should be considered when interpreting the results of our study. Our small sample size limited our ability to discriminate between small but potentially important outcome differences. Although none of the differences we found in clinical outcomes were statistically significant, this may have arisen because of insufficient power to detect 
this level of differences. In addition, the potential exists for unrecognized bias given our nonrandomized design, inclusion of nonshockable rhythms being subject to the discretion of the treating physician, and the possibility of unrecognized factors or confounders associated with patient management and outcome. Our study was performed in an urban metropolitan centre with relatively short transport times; thus, our results may not be generalizable to regions with longer transport times.

The focus of our prehospital $\mathrm{TH}$ intervention on cold IV fluid induction and more aggressive measures to achieve early prehospital cooling may have impacted patients differently as some have suggested that IV fluids may not remain adequately chilled in warm environments. 19,24,25 Our prehospital providers were not equipped with thermometers, and the first recorded temperature on study patients was on hospital arrival. Many of the sicker patients may have experienced autonomic dysregulation, which could have affected their cooling rates. Another consideration was that our prehospital providers' protocol for shivering included discontinuation of TH until arrival at the hospital. We were unable to determine how many patients had their prehospital cooling halted in this manner.

Our study population included those selected as good candidates for TH in our institutional clinical pathway rather than all patients initially resuscitated from OOHCA. The potential for therapeutic momentum via prehospital initiation of cooling may have led to increased patients entering the clinical pathway. This method is generalizable as accrual is more reflective of clinical medicine outside the research setting. We are confident that there was no bias on the part of the prehospital providers in terms of cooling initiation because regardless of ROSC, all patients with OOHCA had prehospital cooling initiated. They were included in the study, following our intention-to-treat analysis, only if they survived to ICU admission with the intention to undergo cooling, regardless of the degree to which the protocol was completed. The initiation of a focused CPR protocol during the study period, with a deemphasis on airway and focus on minimally interrupted chest compressions, may also have affected our findings.

\section{CONCLUSIONS}

OOHCA patients treated with prehospital cooling before arrival at our urban hospital did not have a faster time-to-target temperature or improvement in outcomes compared to patients cooled immediately on ED arrival. Notably, the patients receiving prehospital $\mathrm{TH}$ did experience longer transport times, including arrest-to-ED, ROSC-to-ED, and EMS-arrival-to-ED times. Further research is needed to determine if any benefits exist from prehospital cooling prior to its widespread adoption.

\section{Competing interests: None declared.}

\section{REFERENCES}

1. Mild therapeutic hypothermia to improve the neurologic outcome after cardiac arrest. N Engl 7 Med 2002;346:549-56, doi:10.1056/NEJMoa012689.

2. Bernard SA, Gray TW, Buist MD, et al. Treatment of comatose survivors of out-of-hospital cardiac arrest with induced hypothermia. N Engl f Med 2002;346:557-63, doi:10.1056/NEJMoa003289.

3. Dumas F, Grimaldi D, Zuber B, et al. Is hypothermia after cardiac arrest effective in both shockable and nonshockable patients?: insights from a large registry. Circulation 2011;123: 877-86, doi:10.1161/CIRCULATIONAHA.110.987347.

4. Peberdy MA, Callaway CW, Neumar RW, et al. Part 9: post-cardiac arrest care: 2010 American Heart Association Guidelines for Cardiopulmonary Resuscitation and Emergency Cardiovascular Care. Circulation 2010;122(18 Suppl 3):S768-86, doi:10.1161/CIRCULATIONAHA.110.9710 02 .

5. Kuboyama K, Safar P, Radovsky A, et al. Delay in cooling negates the beneficial effect of mild resuscitative cerebral hypothermia after cardiac arrest in dogs: a prospective, randomized study. Crit Care Med 1993;21:1348-58, doi: 10.1097/00003246-199309000-00019.

6. Nozari A, Safar P, Stezoski SW, et al. Critical time window for intra-arrest cooling with cold saline flush in a dog model of cardiopulmonary resuscitation. Circulation 2006;113:26906, doi:10.1161/CIRCULATIONAHA.106.613349.

7. Zhao D, Abella BS, Beiser DG, et al. Intra-arrest cooling with delayed reperfusion yields higher survival than earlier normothermic resuscitation in a mouse model of cardiac arrest. Resuscitation 2008;77:242-9, doi:10.1016/j.resuscitation. 2007.10.015.

8. Menegazzi JJ, Rittenberger JC, Suffoletto BP, et al. Effects of pre-arrest and intra-arrest hypothermia on ventricular fibrillation and resuscitation. Resuscitation 2009;80:126-32, doi:10.1016/j.resuscitation.2008.09.002.

9. Haugk M, Testori C, Sterz F, et al. Relationship between time to target temperature and outcome in patients treated with therapeutic hypothermia after cardiac arrest. Crit Care 2011;15:R101, doi:10.1186/cc10116.

10. Bruel C, Parienti JJ, Marie W, et al. Mild hypothermia during advanced life support: a preliminary study in out-ofhospital cardiac arrest. Crit Care 2008;12:R31, doi:10.1186/ cc6809.

11. Bernard SA, Smith K, Cameron P, et al. Induction of therapeutic hypothermia by paramedics after resuscitation 
from out-of-hospital ventricular fibrillation cardiac arrest: a randomized controlled trial. Circulation 2010;122:737-42, doi:10.1161/CIRCULATIONAHA.109.906859.

12. Skulec R, Truhlar A, Seblova J, et al. Pre-hospital cooling of patients following cardiac arrest is effective using even low volumes of cold saline. Crit Care 2010;14:R231, doi:10.1186/ cc9386.

13. Kim F, Olsufka M, Longstreth WT Jr, et al. Pilot randomized clinical trial of prehospital induction of mild hypothermia in out-of-hospital cardiac arrest patients with a rapid infusion of 4 degrees C normal saline. Circulation 2007; 115:3064-70, doi:10.1161/CIRCULATIONAHA.106.655480.

14. Castren M, Nordberg P, Svensson L, et al. Intra-arrest transnasal evaporative cooling: a randomized, prehospital, multicenter study (PRINCE: Pre-ROSC IntraNasal Cooling Effectiveness). Circulation 2010;122:729-36, doi:10. 1161/CIRCULATIONAHA.109.931691.

15. Heffner AC, Pearson DA, Nussbaum ML, Jones AE. Regionalization of post-cardiac arrest care: implementation of a cardiac resuscitation center. Am Heart 7 2012;164: 493-501, doi:10.1016/j.ahj.2012.06.014.

16. Jacobs I, Nadkarni V, Bahr J, et al. Cardiac arrest and cardiopulmonary resuscitation outcome reports: update and simplification of the Utstein templates for resuscitation registries: a statement for healthcare professionals from a task force of the International Liaison Committee on Resuscitation (American Heart Association, European Resuscitation Council, Australian Resuscitation Council, New Zealand Resuscitation Council, Heart and Stroke Foundation of Canada, InterAmerican Heart Foundation, Resuscitation Councils of Southern Africa). Circulation 2004; 110:3385-97, doi:10.1161/01.CIR.0000147236.85306.15.

17. Benz-Woerner J, Delodder F, Benz R, et al. Body temperature regulation and outcome after cardiac arrest and therapeutic hypothermia. Resuscitation 2012;83:338-42, doi:10.1016/j.resuscitation.2011.10.026.
18. Studnek JR, Watts JA, Vandeventer S, Pearson D. Assessing the influence of insulation on intravenous fluid infusion temperature. Acad Emerg Med 2012;19:1309-12, doi:10. 1111/acem.12006.

19. Spaite DW, Stiell IG, Bobrow BJ, et al. Effect of transport interval on out-of-hospital cardiac arrest survival in the OPALS study: implications for triaging patients to specialized cardiac arrest centers. Ann Emerg Med 2009;54:248-55, doi:10.1016/j.annemergmed.2008.11.020.

20. Jennett B, Bond M. Assessment of outcome after severe brain damage. Lancet 1975;1:480-4, doi:10.1016/S0140-6736 (75)92830-5.

21. Bernard SA, Smith K, Cameron P, et al. Induction of prehospital therapeutic hypothermia after resuscitation from nonventricular fibrillation cardiac arrest. Crit Care Med 2012;40:747-53, doi:10.1097/CCM.0b013e318237 $\underline{7038}$.

22. Sendelbach S, Hearst MO, Johnson PJ, et al. Effects of variation in temperature management on cerebral performance category scores in patients who received therapeutic hypothermia post cardiac arrest. Resuscitation 2012;83:82934, doi:10.1016/j.resuscitation.2011.12.026.

23. Garrett JS, Studnek JR, Blackwell T, et al. The association between intra-arrest therapeutic hypothermia and return of spontaneous circulation among individuals experiencing out of hospital cardiac arrest. Resuscitation 2011;82:21-5, doi:10. 1016/j.resuscitation.2010.09.473.

24. Murnin M, Sonder P, Janssens G, et al. Patient characteristics, therapy and prognosis determine heat generation in post-cardiac arrest patients receiving therapeutic hypothermia [abstract]. Crit Care Med 2012;40:1-328.

25. Cudnik MT, Schmicker RH, Vaillancourt C, et al. A geospatial assessment of transport distance and survival to discharge in out of hospital cardiac arrest patients: implications for resuscitation centers. Resuscitation 2010;81:518-23, doi:10.1016/j.resuscitation.2009.12.030. 